\title{
Correction to: Multitemporal time series analysis using machine learning models for ground deformation in the Erhai Region, China
}

\author{
Yahui Guo • Shunqiang Hu • Wenxiang Wu • Yuyi \\ Wang $\cdot$ J. Senthilnath
}

Published online: 16 July 2020

(C) Springer Nature Switzerland AG 2020

\section{Correction to: Environ Monit Assess (2020) 192:464 https://doi.org/10.1007/s10661-020-08426-8}

Unfortunately, the name of the corresponding author (Wenxiang $\mathrm{Wu}$ ) was missing in the author group section of the published paper.

The corrected author group is shown above.

Publisher's note Springer Nature remains neutral with regard to jurisdictional claims in published maps and institutional affiliations.

The online version of the original article can be found at https://doi. org/10.1007/s10661-020-08426-8

Y. Guo · W. Wu $(\bowtie)$

Academician Workstation of Zhai Mingguo, University of Sanya, Sanya 572000, China

e-mail: wuwenxiang2018@163.com

$\mathrm{S} . \mathrm{Hu}$

e-mail: hushunqiang8@gmail.com

Y. Guo

e-mail: guoyh@mail.bnu.edu.cn

Y. Guo

Beijing Key Laboratory of Urban Hydrological Cycle and Sponge City Technology, College of Water Sciences, Beijing Normal University, Xinjiekouwaidajie 19, Beijing 100875, China

Y. Wang

e-mail: yuyiwang897@gmail.com

W. Wu

CAS Center for Excellence in Tibetan Plateau Earth Sciences,

Chinese Academy of Sciences (CAS), Beijing 100101, China

J. Senthilnath

Institute for Infocomm Research, Agency for Science, Technology

$\mathrm{S} . \mathrm{Hu} \cdot \mathrm{Y}$. Wang

College of Resource Environment and Tourism, Capital Normal University, Beijing 100048, China 